\title{
Ulrich Hohoff
}

\section{Universitätsprofil und Bibliotheksprofil}

\section{Vorbemerkung}

Seit etwa drei Jahren verstarken sich in Wissenschaft und Wissenschaftspolitik in Deutschland Tendenzen, den Universităten (einschlieBlich ihrer Bibliotheken) ein klareres Profil zu geben.

Erstens fordern Politiker zunehmend, die Universităten sollten stărker an die Öffentlichkeit gehen und ihre Leistungen in Forschung und Lehre deutlicher herausstellen, damit der Bürger sehen konne, welche Früchte die hierfür aufgewendeten Steuermittel trügen. Sie sollen sich als Teil der Gesellschaft legitimieren. Außerdem fordert die Politik finanzielle Transparenz und mehr Leistungsanreize als Voraussetzungen für eine profilierte Universităt.

Zweitens zwingen sinkende Etats die Bundesländer zu stărkerer Koordinierung von Studiengången und Forschungseinrichtungen an den Universitäten. Ganze Fakultăten werden umstrukturiert, zahlreiche wissenschaftliche Einrichtungen neu ausgerichtet, geschlossen oder zusammengelegt. Die tiefsten Einschnitte treffen das Land Berlin. Die Verănderungen tragen angeblich dazu bei, jeder Universität ihr eigenes Profil zu geben. Studienangebote und Forschungsschwerpunkte im Land sollen sich voneinander abheben, ihre Vielfalt soll sichtbar werden.

Drittens zielt die beginnende Hochschulreform darauf ab, den Universitäten mehr Autonomie in fachlichen, finanziellen und organisatorischen Fragen zu geben. Die Universităten kơnnten also im Prinzip Forschung und Lehre weitgehend kraft eigener Entscheidung weiterentwickeln.

Beide Entwicklungen, ein gestiegenes Kostenbewußtsein (u.a. bei der Frage, welche Leistungen unter Einsatz welcher Mittel zu erreichen sind) und ein Zuwachs an Autonomie, sollten einen Zielfindungsprozeß der Universităt in Gang setzen. An dessen Ende würden die Universităten in Forschung und Lehre ein schärferes Profil als heute haben, wenn sie ihre Chancen nützen. Die finanziellen Zwănge und der gesellschaftspolitische Rechtfertigungsdruck, die heute auf den Universităten lasten, hătten am Ende doch inhaltlich etwas bewirkt: Klarheit über die eigenen Ziele, ein zukunftsorientiertes Handeln und ein gestărktes Selbstbewußtsein. Aber der Weg dorthin wird schwierig sein.

An einigen Orten wurden die Entwicklungs- und Planungskommissionen bereits beauftragt, diesen Proze $B$ anzugehen. Meinungsbildung und Diskussion zum 
Thema werden sich über Jahre erstrecken. Die Universitătsbibliotheken als zentrale Einrichtungen ihrer Universitaten sollten sich in den Prozeß der Profilierung ihrer Universitat einschalten bzw. daran beteiligt werden. Ihre relative Autonomie in der Universitat macht sie zu einem starken Partner, der jenseits fachlicher Partikularinteressen wichtige Schwerpunkte beitragen kann.

Die folgenden Überlegungen nehmen eingangs zum Begriff „Profil“ Stellung. Sie pladieren dann für einen eigenen Beitrag der Universitatsbibliothek zum Profil ihrer Universität. Anschließend zeigen zwei Szenarios zu einem Ausschnitt der Problematik, welche erheblichen Folgen für die universităre Literaturversorgung ein profilorientierter Universitătsbetrieb zeitigen kann.

\section{Was ist ein Universitätsprofil?}

Der Anspruch an ein Universitätsprofil ist hoch: Es soll die Eigenart und Strategie der Universităt im Unterschied zu anderen Universităten deutlich machen. $\mathrm{Ob}$ anstelle von ${ }_{n}$ Profil $^{4}$ ein anderer Begriff eingesetzt wird, ist nicht entscheidend. Das ist nicht im Sinn einer Werbeschrift gemeint, die etwa den Ist-Stand mit blumigen Worten und schonen Bildern schilderte. Es ist eher einem detaillierten Firmenprofil verwandt, das zeigt, welche Ziele die Firma sich setzt, in welche Produktlinien sie ihre Ziele umsetzt und wie sie im Wettbewerb innerhalb ihrer Branche einzuordnen ist.

Um solche Aussagen treffen zu können, müssen - bei allen Unterschieden zwischen Universităten und Wirtschaftsbetrieben - auch Universităten einige Voraussetzungen erfüllen: Es muß gemeinsame Ziele geben, es muß Absprachen über deren Umsetzung geben, und die eigene Position im Umfeld weiterer Wissenschaftseinrichtungen mub bestimmt worden sein.

Schon an diesem Punkt wird die strategische Bedeutung eines Universitătsprofils deutlich Es setzt eine Analyse des Ist-Standes voraus, es entsteht als Ergebnis eines Zielfindungsprozesses und es formuliert das Selbstverstăndnis, die Angebote und die Zielrichtung der Universităt. Bei der Arbeit am eigenen Profil ist zugleich eine mittelfristige Planung für die Universităt zu leisten. Das Profil enthalt das Leitbild und das Selbstbild der Universităt. Es verdeutlicht ihre Stärken und Schwerpunkte, die in einen Planungsrahmen eingebettet sind.

Ein unverwechselbares Profil einer Universitat kann daher nicht ein rasch erstelltes Papier von nur momentaner Bedeutung sein. Es kann nur am Ende eines längeren und grundsatzlichen Planungs- und Diskussionsprozesses über die ganze Universitat erstellt werden. Einerseits stellt es die Bereiche Forschung und Lehre dar, andererseits die hierfür aufgebauten Dienstleistıngen. Wie die publi- 
zierten Ranglisten von Universităten zeigen, die auch in Deutschland in Mode gekommen sind, werden Fragen der Literatur- und Informationsversorgung grundsätzlich mit berücksichtigt.

Da die Universităten ihr Lehr-, Forschungs- und Dienstleistungsprofil selbst erarbeiten werden, besteht zwar die Gefahr, daB es nur den Status quo festschreibt und die Abhangigkeit von Gremienmehrheiten widerspiegelt. Doch schafft Druck von außen den $Z$ wang, vorausschauend zu planen. Denn andere Universităten werden sich als Partner und/oder als Konkurrenten ebenfalls profilieren, um ihre Chancen zu nützen.

Das Profil der Universităt muß, wie wir sahen, aus Überlegungen zur Weiterentwicklung hervorgehen. Mit anderen Worten: Als profilfăhig bzw. profilprăgend wird zu beschreiben sein, was in der Entwicklungsplanung der Universităt als zukunftstrăchtig gilt. Die konkrete Ausformung eines Universitătsprofils wird von Bundesland zu Bundesland und von Universităt zu Universităt unterschiedlich ausfallen. Einige Merkmale gelten unabhăngig davon für jedes Universitătsprofil:

- Ergebnis eines Zielfindungsprozesses der Universităt und Einbettung in deren strategische Entwicklungsplanung

- Formulierung der spezifischen Stărken und Schwerpunkte in Forschung, Lehre und Dienstleistung

- Unterscheidung zwischen Făchern/Schwerpunkten, die das Profil prăgen und jenen, die es nicht prăgen

- Fä́cher/Schwerpunkte, die das Profil prăgen, werden besser ausgebaut und ausgestattet sein als die übrigen.

\section{Beiträge der Universitätsbibliothek zum Universitătsprofil}

Der Planungsprozeß und das Universitătsprofil als sein Ergebnis wirken beide auf die UB als zentrale Dienstleistungseinrichtung der Universităt ein. Sie ist ein wichtiger Standortfaktor einer Universitat, denn nicht nur die aktuellen Neuerwerbungen sind die Voraussetzung für hochwertige Lehre und Forschung. Auch Größe und Art vorhandener Buchbestande in den Geistes- und Sozialwissenschaften sowie die Anzahl der gehaltenen bzw. am Ort zugănglichen Fachzeitschriften in Naturwissenschaften, Medizin und Technik sind für Dozenten häufig wesentliche Kriterien bei der Entscheidung, ob eine Bewerbung eingereicht wird, ob ein Ruf angenommen, abgelehnt oder mit Bedingungen verknüpft wird (bessere Ausstattung mit Fachliteratur für Forschungsschwerpunkte etc.). 
Die UB kann den schwierigen Diskussionsprozeß für ein entstehendes Universitătsprofil mit ihrem Bestand „unterfüttern". Denn über lange Zeitrăume hinweg haben Universitătsbibliotheken mit betrăchtlichen Mitteln in sich geschlossene Sammlungen gezielt aufgebaut, die in der Regel auch als solche erschlossen oder zumindest dokumentiert sind. Hier sind vor allem Sammlungen mit regionalen oder thematischen Schwerpunkten, Sammlungen zu Spezialthemen, Nachlaßbibliotheken, übernommene Bibliotheken anderer Einrichtungen, Schenkungen und museale Sammlungen zu nennen. Hinzu treten geschlossen übernommene Bibliotheken (von Privat, von Vereinen, von offentlichen oder wissenschaftlichen Einrichtungen etc.) und die klassischen Gebiete Handschriften, alte Druckschriften (bzw. Zeitschriften) und Autographen.

Sie bieten ein von Universităt zu Universităt anders geprägtes Potential für die Forschung, auf das ein Forschungsprofil zurückgreifen sollte. Dann prăgt der UB-Bestand es mit. Je starker Forscher schon heute mit diesen spezifischen Sammlungen der UB arbeiten, desto eher wird dies Vorgehen die Zustimmung der Universităt finden.

Erfahrungen aus den sog. Buchwissenschaften zeigen, daß es für die Universităt ein Gewinn ist, wenn sie eine Professur - oder ein ganzes Institut - an Bestandsschwerpunkten der UB ausrichtet. Der Forscher wird dank der jeweiligen Sammlung sehr gute Arbeitsbedingungen vorfinden. Die Sammlungen ihrerseits sind oft nach Absprache mit Wissenschaftlern der Universităt aufgebaut oder angekauft worden, so daß der Neuankommling an eine Forschungstradition mit wichtigem überregionalen Renommee anknüpfen kann. Nicht selten sind sie es, die ihn bewegen, den Ruf anzunehmen.

Ein gutes Beispiel zu diesem Thema ist die Oettingen-Wallersteinsche Bibliothek in der UB Augsburg, die das Land Bayern vor 18 Jahren angekauft hat.

Die UB Augsburg erbielt damit als in sich geschlossenen Altbestand eine über Jahrhunderte sorgfaltig aufgebaute fürtliche Bibliothek, die einen wertvollen Altbestand von 117.000 Bänden, 1.500 Handschriften, 1.000 Inkunabeln und 1.787 Musikhandschriften umfabt und regionale Bezüge zu Bayerisch-Schwaben aufweist. Diesen Schatz hat die UB Augsburg aus eigener Kraft maschinenlesbar katalogisient und uber Ausstellungen und Publikationen rasch zugănglich gemacht.

Die Oettingen-Wallersteinsche Bibliothek in der UB Augsburg und die reichen Altbestande der Staats- und Stadibibliothek Augsburg gaben einige Jahre spater den Ausschlag fiur die Entscheidung, an der Universität Augsburg ein interdisziplinăres „Institut für Europaische Kulturgeschichte ${ }^{a}$ ins Leben zu rufen, das die 
Werke nach und nach wissenschaftlich auswertet. Die UB Augsburg stellte die Sammlung auch Lehrstühlen verschiedener Disziplinen vor, die in Projekten, in wissenschaftlichen Veranstaltungen und in Ausstellungen zusammen mit der UB fachlich interessante Teile des Bestandes bearbeiteten. Die Mühe hat sich für den Bibliotheksleiter, Dr. Frankenberger, gelohnt, denn die UB Augsburg hat exemplarisch vorgeführt, wie ein Haus mit bedeutendem Bibliotheksbestand Forschung stimulieren kann.

Wie das Beispiel verdeutlicht, reicht es nicht aus, eine besondere Sammlung nur zu besitzen. Die Bibliothek muß sie auch komfortabel erschließen und der innerund außeruniversitären Öffentlichkeit in Eigeninitiative bekanntmachen. Neben Sammlungen dieses Typs, die ein Universitătsprofil schărfen können, kommen natürlich spezifische Bestände am Ort in Frage, die aufgrund eines gesetzlichen Auftrags (Pflichtexemplar) oder aus der Einbindung in die bundesweite Kooperation wissenschaftlicher Bibliotheken entstanden sind (Sondersammelgebiete).

In Zukunft werden neben gedruckten Sammlungen elektronisch gespeicherte Bibliotheken, die von der UB aufgebaut werden oder zu denen sie Zugang verschaff, ein wichtiges Spezifikum im bibliothekarischen Angebot werden, das sich ebenfalls in das Universitătsprofil einbringen läßt. Schwerpunkte kőnnen z.B. sein: die Rolle der UB als Kompetenzzentrum in Digitalisierungsfragen, ein umfangreicher Zugriff auf entfernt gespeicherte Volltexte, die rasche Lieferung der Volltexte über Datennetze, neue Rollen der UB als elektronisches Textzentrum ihrer Universität für die Forschung oder als Zentrum für multimediales Lernen.

3. Szenario I: Veränderungen der Literaturversorgung von Fächern, die das Universitātsprofil prägen

Zu Beginn ihrer Arbeit am Profil von Forschung, Lehre und Dienstleistung muß die Universităt u.a. die 'profilprăgende Einheit' definieren. Vorrangig dürften hierfür die Wissenschaftsfächer in Frage kommen. Auch größere übergreifende Forschungsthemen oder bestimmte Institute mit klarer inhaltlicher Ausrichtung und einzelne Studienangebote konnen das Profil prägen. Das Überschreiten der engen Grenzen von Fakultăten, Instituten oder Berufungsgebieten hin zu interdisziplinăren Forschungen über größere Themengebiete wird sich mit Sicherheit verstårken, weil für die Mehrheit wichtiger Probleme nur so Lösungen zu finden sind. Im Folgenden dient noch die überholte Făcherstruktur als Beispiel.

In einem weiteren Schritt muß die Universităt festlegen, welche Fächer, die an der Universităt vertreten sind, das Profil in der Forschung und/oder der Lehre prăgen bzw. prägen sollen. Dieser Schritt hat weitreichende Folgen: Profilprä- 
gende Făcher werden von andern unterschieden, die als nicht so entscheidend gelten. Diesen Unterschied schreibt die Planung mittelfristig fest.

Eine Stufe unterhalb der profilprăgenden Făcher werden Făcher stehen, die unentbehrlich sind, auch wenn sie nicht das Profil ausmachen (z.B. Wirtschaftsund Rechtswissenschaften, Philologien, traditionell gelehrte Făcher etc.).

Diese Abstufiung läbt sich erweitern.

Sicher ist, dab die Einstufung eines Faches nach seiner Wichtigkeit für das Profil der Universităt sich künftig auf die Zuteilung der verfügbaren Mittel auswirken wird. Schon immer gab es Fächer, die Vorteile vor anderen genossen oder sich der besonderen Unterstützung der Universitătsleitung erfreuten. Entscheidend am Prozeß der Profilierung für den Wettbewerb ist aber, daß eine Rangfolge beabsichtigt ist, in der universitären Öffentlichkeit erarbeitet und zum verbindlichen Leitbild erhoben wird. Sie ist daher in hohem Maße verbindlich.

Ist das Universitătsprofil erarbeitet und veröffentlicht, dann mũssen den guten Ruf einer Universităt vornehmlich die profilprägenden Fächer rechtfertigen und verantworten.

Sie werden in der Forschung stark ausdifferenziert sein. Sie bekommen Personalund Ausstattungswünsche leichter erfüllt als andere. Das gilt auch für die Versorgung mit Büchern, Zeitschriften und elektronischen Informationen. Es wird genügend Geld für spezielle Forschungsliteratur zur Verfügung stehen, ebenso für eine ausreichende Anzahl an Lehrbüchern und Studienliteratur. Falls ihnen eigene dezentrale Bibliotheken zugeordnet sind, kommen hierfür Personal, Sachmittel, Gerăte, Netzanschlüsse usw. hinzu. Für Benufungs- oder Bleibeverhandlungen von Professoren stehen Profil-Făchern höhere Summen zur Verfiigung als den übrigen. Es wird bei ihnen mehr Drittmittelprojekte geben, für die wiederum Bibliotheksmittel nơtig sein kơnnen. Dasselbe gilt für Gastprofessuren und andere Sonderleistungen.

Diese Vorteile kőnnen auch der Literaturversorgung dieser Făcher zugute kommen. Es gibt aber eine Hürde: Ein profilbildendes Fach wird eher als andere über Globalzuweisungen verfügen, damit Flexibilităt erhalten bleibt. Die Frage, wofür Mittel in welcher Hohe eingesetzt werden, entscheidet dann das Leitungsgremium der Einrichtung, in der das Fach angesiedelt ist.

Diese Situation kann die bibliothekarische Versorgung, die mit anderen Ausgabezwecken konkurriert, in den Hintergrund drangen. Die für Bücher, Zeitschriften und elektronische Medien notwendige bzw. im Vorjahr ausgegebene Summe kann z.B. auch für die befristete Anstellung von Personal, für wissenschaftliche 
Gerăte oder als Eigenanteil bei Projekten eingesetzt werden. Die Folge wäre stetige Unsicherheit über die Höhe verfügbarer Mittel pro Fach, zumal deren „Umwidmung" für andere als Bibliothekszwecke auch mitten im Haushaltsjahr mogglich ist. Diese Bedingungen erschweren einen geplanten, kontinuierlichen und verantwortlichen Bestandsaufbau beträchtlich.

Um sie zu vermeiden, muß die UB bei Etatfragen um Mittel zugunsten des Wissenschaftsfaches bzw. -schwerpunkts mitsprechen und gute Argumente vortragen. An Entscheidungen über die Mittelverteilung muß sie beteiligt sein. Zumindest ein jăhrlicher Sockel ist als Garantiebetrag notwendig, um Fortsetzungsverpflichtungen, elektronische Medien und den Kauf von Studienliteratur und von wichtigen Monographien zu sichern.

Ein Fach, welches das Universitătsprofil mitbestimmt, wird einerseits die erwähnten Vorteile genießen. Andererseits wird es sich regelmäBig strengen Qualităts- und Leistungskontrollen stellen müssen, damit die Universităt in diesem Fach Spitzenleistungen anbieten kann.

Nach den bisher publizierten rankings zu urteilen, gelten die Geschwindigkeit der Bereitstellung und die Breite des Angebots neben Benutzungsfragen als wesentliche Faktoren fuir die Qualităt der Literaturversorgung. Hier kommen folgende Indikatoren ins Blickfeld: Höhe der Bibliotheksmittel für das Fach und pro Professor, Höhe der Ausgaben für die Lehrbuchsammlung und erworbene Titel pro Student; Öffnungszeiten; Größe des Freihandbestands; Dauer der Fernleihe; Dauer des Geschăftsgangs vom Eintreffen im Haus bis zur Bereitstellung; Personal- und Sachmittelausstattung, bibliothekstechnische Ausstattung.

Die Universităt kann in ihrem Lehr- und Forschungsprofil unterscheiden zwischen Făchern, die in Lehre und Forschung gleichermaßen profilprăgend sind, und anderen, auf die das nur in der Lehre oder nur in der Forschung zutrifft. Letzteres gilt in der Regel für sogenannte Orchideenfächer und für Lehrstühle, die zur Entwicklung und Förderung der Region notwendig sind. Umgekehrt könnte die Ausbildung in einem "Massenfach" wie Betriebswirtschaft oder Erziehungswissenschaft überregional als vorbildlich gelten, auch wenn in diesen Fächern kaum geforscht würde. Wenn die Universităt Studenten für profilprăgende Fächer anwerben will, dann müssen vornehmlich sie attraktiv ausgestattet sein. Da die Studentenzahl und die Ausbildungskosten pro Student auch Richtwerte zum Bemessen von Haushaltsbudgets sind, bleiben im Hinblick auf das Profil der Lehre teure und studentenreiche Ausbildungsgănge tendenziell erhalten.

Bei Erwerbungen in profilprägenden Făchern sind Lehrbücher und Studienliteratur in großer Breite und Titelzahl zu ergănzen, damit keine Wartezeiten entste- 
hen, die zu Studienverzőgerungen führen könnten. In „Massenfächern“ kann das bei bestimmten Titeln zu einer bisher vermiedenen hohen Exemplarzahl führen. Die Universităten werden auch fordern, daß vorrangig für Profilfächer zumindest nevere und hăufig benutzte Literatur direkt am Regal zugånglich gemacht wird, wie es in den einschichtigen Bibliothekssystemen und in Institutsbibliotheken zweischichtiger Systeme üblich ist.

Die Ausdifferenzienung der Făcher im Zuge der Profilienung ăndert nichts am gesetzlichen Versorgungsauftrag der Universitătsbibliotheken. Sie beeinflußt aber die Rangfolge, in der die Aufgaben zu erfillen sind. Da profilprägende Fächer Vorzeigefächer für die Entwicklung und das Image der Universităt sein werden, wird die Universitat dafür Sorge tragen, daß die UB vorrangig sie mit Literatur und Medien versorgt.

\section{Szenario II: Veränderungen der Literaturversorgung bei anderen Fächern}

Wenn die Universitat nach ihrem Profil arbeitet, werden sich die Ausbauziele und das Bestandsniveau im Bibliothekssystem von Fach zu Fach ausdifferenzieren nach dem Prinzip: Grundversorgung für alle Făcher, Spitzenversorgung nur für einige.

Für manche Wissenschaftspolitiker (und auch Bibliothekare) bedeutet das, von einer Leitidee Abschied za nehmen, nămlich von der gleichmăßig intensiven Versorgung aller Făcher an der Universităt mit Büchern und Zeitschriften. Sie ist schon seit Jahren aus finanziellen Gründen nicht mehr realisierbar. Wenn die Ökonomisierung der Universităten sich fortsetzt, wird die Wissenschaftspolitik dieses Ziel aufgeben müssen. Das Forschungspotential der Universităt sowie die Studien- und Lehrangebote werden sich ăndern, wenn der Wettbewerb der Fächer um Ressourcen sich verschărft. Făcher, die nicht das universităre Profil prăgen, werden zwei Effekte spüren, die auch auf die UB durchschlagen.

Erstens wird die Universităt für profilprăgende Făcher zusătzliche Mittel benōtigen. Nach heutiger Finanzlage bedeutet das, dab diese Summe bei anderen Fächern einzusparen ist. Die Mittelverteilungsdiskussion wird schărfer.

Zweitens mussen, wenn die Universität selbst über Gründung und Auflösung ihrer Einrichtungen und über ihre Studienangebote bestimmen kann, jene Fácher, die im Bundesland mehrfach angeboten werden, klare Profile erhalten. Făcher, Abteilungen und Studienrichtungen innerhalb einer Universităt konnen geschlossen oder zusammengelegt werden. Studienrichtungen, die heute an zwei Universităten gelehrt werden, könnten zusammengelegt werden. Momentan weniger gefragte Fachrichtungen werden newen Schwerpunkten weichen. 
Die UB wird dann die Erwerbungstătigkeit für jahrzehntelang aufgebaute Teilbestände von z.T. speziellster Fachliteratur für bestimmte Făcher stark einschrănken oder ganz einstellen müssen. Ausgaben für Bücher, Zeitschriften und andere Medien werden auch bei Fâchern, die nicht das Universitătsprofil prägen, in Konkurrenz zu anderen Ausgabezwecken treten. Um so wichtiger wird es, für sie ein Konzept zur Finanzienung eines Grundbestands an Fachliteratur zu haben, das transparente und akzeptable Berechnungskriterien verwendet.

Die Probleme liegen von Fach zu Fach anders. Aber typisieren låßt sich in einer Erwerbungskonzeption durchaus. Bei der Literatur eines Wissenschaftsfaches lassen sich grob die Kategorien Allgemeine und Grundlegende Literatur, Literatur für die Forschung und Literatur für Studium/Lehre unterscheiden. Der erstgenannte Posten ist prinzipiell für alle Nutzer des Fachs da, der zweite für die Wissenschaftler und fortgeschrittene Studenten, der dritte enthalt Fachliteratur für Studenten in der ersten Studienhälfte. Die Beschaffungsintensităt sollte je nach Erscheinungsland definiert sein. Der Geldbedarf läßt sich nach Bandzahlen und Durchschnittspreisen errechnen.

Die Sicherung eines nennenswerten Etatanteils für Lehrbücher erfordert Fingerspitzengefuihl. Denn die Făcher sollen attraktiv bleiben. Je knapper die Erwerbungsmittel sind, desto eher sehen aber Wissenschaftler den Vorrang beim eigenen Bedarf für die Forschung. Hier sind die Studenten selbst gefordert, aber auch die UB in ihrer Rolle als Anwalt studentischer Benutzer.

Beim Bestandsaufbau schlăgt dem Bibliothekssystem wie immer bei knappen Ressourcen die Stunde der Koordinienung. Koordinierung meint nicht nur den Versuch, Erwerbungsabsichten abzustimmen, sondern das Prinzip, nur nach Koordinierung $\mathrm{zu}$ bestellen, also nichts zu bestellen, was koordinierter Erwerbung widerspricht. In zweischichtigen universităren Bibliothekssystemen wird es Chancen geben, die Literaturversorgung straffer zu organisieren und wirtschaftlicher zu arbeiten. Damit das gelingt, sollten Universitătsleitung und UB Lösungen erarbeiten, bei denen die UB die Organisationsgewalt auch über dezentrale bibliothekarische Arbeiten erhält. Die Verantwortung für Koordinienungsentscheidungen im Einzelfall muß bei der UB liegen. Sonst kann sie ihre Aufgabe als zentrale Dienstleistungseinrichtung für die Universităt nicht erfüllen.

Zeitschriftenabonnements und teure Werke sollten in der Regel in einem Exemplar beschaff werden oder den elektronischen Zugriff mitnutzen. Titel, auf die mehrere Făcher zugreifen, müssen am zentralen Standort zugănglich sein. Titel, deren Bezug sich an der Universităt und mit wissenschaftlichen Bibliotheken am Ort nicht mehr koordinieren läbt, sollen auf Landesebene koordiniert werden. Bei weniger teuren Zeitschriften kann die Kündigung des Abonnements der Univer- 
sităt aber bedeuten, daß bisherige Erwerbungskosten nur verlagert werden, nămlich auf Besorgungskosten via Fernleihe.

Schließlich muß eine UB auch bei der Arbeit nach einem Universitătsprofil den Bedarf weiterer Benutzergnuppen und Sammelgebiete abdecken, die nur bedingt die Universităt berühren.

Finanziell abzusichern sind natürlich Erwerbungsaufgaben einer UB, die tiber ihre Universităt hinaus reichen und auf regionalen oder überregionalen Vereinbarungen zur Zusammenarbeit im wissenschaftlichen Bibliothekswesen beruhen (z.B. Sondersammelgebiet, Sammelschwerpunkt, Pflichtexemplar, Spezialsammlung). Oft sind sie im Haushalt nicht separat veranschlagt.

Eine UB kann trotz starker Nachfrage der Universităt nach Studien- und hochspezieller Forschungsliteratur nicht ganz auf den Kauf von Werken verzichten, die mehrere andere Benutzer brauchen. Die Erwerbung sollte z.B. weiterhin Benutzerwinsche erfillen.

Die wichtigste Benutzergruppe neben den Mitgliedern der Universităt sind Benutzer aus Stadt und Region. Universitätsmitglieder übersehen das manchmal oder erkennen nicht an, daß diese Gruppe in Teilen ihren spezifischen Literaturbedarf hat und auch für den Ruf der UB in der Region außerordentlich wichtig ist, weil sie viele Studienabsolventen der Universität einschlieBt. Sie ist oft die zweitgroßte Benutzergruppe, hat aber leider keine Lobby. Der Literaturversorgungsauftrag jedoch schließt sie ein. Denn die Hochschulgesetze halten fest, daß die UB auch der "beruflichen Fort- und Weiterbildung" diene. Damit ist gemeint, daB die UB diese Gruppe zur Benutzung zulassen muB, aber auch, daß Literatur für diesen Bedarf erworben wird, wenngleich in geringem Umfang. Wegen dieses Auftrags darf es nicht geschehen, daB eine UB z.B. ihre Dienste für Einzelbenutzer aus Stadt und Region, für Behơrden oder für mittelstăndische Betriebe aus Geldmangel bei der Erwerbung einstellen muB. 